\title{
KOMMENTAR
}

\section{Was tun bei Inflations- und Rezessionsängsten? \\ Jan Priewe}

Die Inflationsrate ist in Deutschland mit 3,4\% so hoch wie seit 15 Jahren nicht mehr, in der Eurozone lag sie Mitte 2008 bei 4,0 \%, in den USA sogar bei 5,0\%. Es sind vor allem die Energie-, Rohstoffund Nahrungsmittelpreise, die die Inflation treiben. In der Eurozone machen diese Produkte etwa $30 \%$ des Warenkorbs aus, in Deutschland gut ein Fünftel. Die sogenannte Kerninflation, also Energieund Nahrungsmittelpreise herausgerechnet, liegt indessen bei nur $1,8 \%$ im Euroraum, damit unter der Zielmarge der Europäischen Zentralbank (EZB) von "nahe, aber unter 2,0\%".

Das wirft eine ganze Reihe von Fragen auf: Ist die neue Inflation gefährlich, oder reagiert die EZB panisch? Was sollte die Tariflohnpolitik tun? Warum ist die US-Geldpolitik ganz anders als die der EZB - die Fed (Federal Reserve Bank of New York) hat den Leitzins auf 2,0 \% gesenkt, die EZB gerade im Juli auf 4,25\% hochgeschraubt. Ist die Inflation oder die restriktive Geldpolitik das Problem?

Eigentlich ist ein bisschen Inflation, auch wenn es mal etwas mehr als $2 \%$ sind, für Konjunktur und Beschäftigung nicht so dramatisch, wenn sie sich nicht beschleunigt. Das tut sie aber, wenn alle sich daran gewöhnen, also höhere Inflationserwartungen entwickeln und daran ihr Verhalten ausrichten. Preis-Lohn-Spiralen entstünden, die die Inflation beschleunigen. Genau diese sogenannten Zweitrundeneffekte befürchtet die EZB. Tritt Inflationsbeschleunigung ein, muss die Zentralbank früher oder später auf die Bremse treten, also mit hohen Zinsen eine „harte Landung“, sprich Rezession, herbeiführen. Gewerkschaften sind besser beraten, wenn sie aus gesamtwirtschaftlicher Vernunft den Teuerungsausgleich in etwa auf die Zielinflationsrate beschränken und auf mehr Reallohn durch weniger Inflation hoffen. Setzen sie jedoch vollen Inflationsausgleich zusätzlich zum Produktivitätsanstieg durch, werden die Unternehmen diesen in die Preise überwälzen. Die Lohntüte wird real nicht voller, aber die EZB wird hart reagieren. Das ist klar die schlechtere Option, die zu Beginn der 1970er Jahre gewählt worden war.

Für die unteren Einkommensschichten und auch die Masse der Arbeitnehmer gibt es keinen Grund für Inflationstoleranz. Gerade die jetzige Inflation trifft Geringverdiener überproportional, weil sie mehr als der Durchschnitt der Haushalte für Nahrungsmittel und Energie ausgeben. Sparer, deren Sparzins nicht der Inflation angepasst ist, verlieren ebenfalls, ebenso Bezieher fester, politisch gesetzter Einkommen. Aber wenn Preisstabilität durch geringere Beschäftigung erkauft würde, wäre unterm Strich wenig gewonnen.

Wie soll die EZB reagieren? Zunächst kann sie gegen die neue globale Inflation der Rohstoff- und Nahrungsmittelpreise gar nicht viel tun. Sie könnte allerdings die Preise anderer Güter kompensatorisch senken. Dies erfordert aber eine deutlich restriktivere Geldpolitik, die richtig weh tut. Diesen Kurs scheint bedauerlicherweise die EZB gehen zu wollen, im Gegensatz zur US-Fed. Gewiss, die USKonjunktur ist derzeit schwächer als die europäische, und die Prob- leme der Finanzkrise wiegen dort schwerer. Aber deswegen muss die EZB doch die ohnehin kippelige Konjunktur in Europa nicht bremsen. 2008 wird seitens der Zentralbank nur noch ein Wachstum des Bruttoinlandsprodukts von 1,6\% erwartet, 2009 nur noch 1,3\%. In den guten Jahren 2006 und 2007 waren es noch 2,8 \% bzw. 2,7 \%. Die Arbeitslosigkeit war in Euroland von 2004 bis zu Beginn dieses Jahres gesunken, jetzt wird sie wieder steigen. Wieder einmal leben wir in Europa unter unseren Verhältnissen, gemessen an dem, was angebotsseitig möglich wäre, wenn es an Nachfrage nicht mangeln würde.

Zwar ist die Inflation in Euroland zu hoch, aber die Beschleunigungsgefahr ist bislang gering geblieben. Die Lohnstückkosten sind trotz guter Konjunktur der vergangenen Jahre nur leicht gestiegen. Selbst die EZB-Philosophie sagt, dass die Zielinflation von $2 \%$ mittelfristig angestrebt werden soll, kurzfristige Abweichungen seien hinzunehmen. Sie könnten auch gar nicht mit den Mitteln der Geldpolitik bekämpft werden, weil die Wirkungsverzögerungen bei über einem Jahr liegen. Die Inflationsprognose der EZB erwartet für das Jahr 2008 insgesamt 3,4 \%, 2009 2,6\% und 2010 2,1 \% Inflation. Demnach ist der Höhepunkt der Teuerung bereits überschritten. Dies ist plausibel, denn bei schwächerer Weltkonjunktur geben die konjunkturreagiblen Rohstoff- und Energiepreise nach. Ein Teil des aktuellen Preisniveauanstiegs ist Folge der Globalisierung, den müssen wir nolens volens hinnehmen. Ein anderer Teil ist ökologisch sinnvoll, weil er die Knappheit fossiler Energie reflektiert. Auch diese Komponente müssen wir akzeptieren, und so sollte es auch die EZB halten.

Hausgemachte Inflation durch Zweitrundeneffekte kann die EZB freilich nicht tatenlos hinnehmen. Aber aggressive Nominallohnpolitik auf breiter Front ist, wie gesagt, einfach nicht in Sicht. Bei einer labilen Konjunktur war daher die Zinsanhebung um 25 Basispunkte im Juli ein falsches Signal. Etwas Zinssenkung wäre besser gewesen, allerdings nicht unbedingt auf das US-Niveau. Überzieht die Geldpolitik die Restriktion, wäre es Aufgabe der Fiskalpolitik, antizyklisch gegenzusteuern - etwa mit höheren Staatsausgaben für Bildung oder zur Entlastung inflationsgeschädigter Geringverdiener. Erst recht, wenn die gesamtwirtschaftliche Nominallohnentwicklung trotz Inflation moderat verläuft und eine deutliche Nachfragelücke absehbar ist.

\footnotetext{
Dr. Jan Priewe, Professor für Volkswirtschaftslehre an der Fachhochschule für Technik und Wirtschaft (FHTW) Berlin. Arbeitsschwerpunkte: Makroökonomie, Wirtschaftspolitik, Ökonomie von Entwicklungsländern. e-mail: priewe@fhtw-berlin.de
} 